\title{
Pelvic incidence-lumbar lordosis mismatch predisposes to adjacent segment disease after lumbar spinal fusion
}

\author{
Dominique A. Rothenfluh • Daniel A. Mueller • \\ Esin Rothenfluh $\cdot$ Kan Min
}

Received: 21 May 2013/Revised: 2 July 2014/Accepted: 2 July 2014/Published online: 14 July 2014

(c) Springer-Verlag Berlin Heidelberg 2014

\begin{abstract}
Purpose Several risk factors and causes of adjacent segment disease have been debated; however, no quantitative relationship to spino-pelvic parameters has been established so far. A retrospective case-control study was carried out to investigate spino-pelvic alignment in patients with adjacent segment disease compared to a control group. Methods 45 patients (ASDis) were identified that underwent revision surgery for adjacent segment disease after on average 49 months (7-125), 39 patients were selected as control group (CTRL) similar in the distribution of the matching variables, such as age, gender, preoperative degenerative changes, and numbers of segments fused with a mean follow-up of 84 months (61-142) (total $n=84$ ). Several radiographic parameters were measured on preand postoperative radiographs, including lumbar lordosis measured (LL), sacral slope, pelvic incidence (PI), and tilt. Results Significant differences between ASDis and CTRL groups on preoperative radiographs were seen for PI $\left(60.9 \pm 10.0^{\circ}\right.$ vs. $\left.51.7 \pm 10.4^{\circ}, p=0.001\right)$ and $\mathrm{LL}$ $\left(48.1 \pm 12.5^{\circ}\right.$ vs. $\left.53.8 \pm 10.8^{\circ}, p=0.012\right)$. Pelvic incidence was put into relation to lumbar lordosis by calculating the difference between pelvic incidence and lumbar lordosis $\left(\triangle \mathrm{PILL}=\mathrm{PI}-\mathrm{LL}, \mathrm{ASDis} 12.5 \pm 16.7^{\circ}\right.$ vs. CTRL $\left.3.4 \pm 12.1^{\circ}, p=0.001\right)$. A cutoff value of $9.8^{\circ}$ was determined by logistic regression and ROC analysis and patients classified into a type $\mathrm{A}\left(\Delta \mathrm{PILL}<10^{\circ}\right)$ and a type $\mathrm{B}$
\end{abstract}

D. A. Rothenfluh · D. A. Mueller · E. Rothenfluh · K. Min Department of Orthopaedics, Uniklinik Balgrist, University of Zurich, Zurich, Switzerland

D. A. Rothenfluh $(\bowtie)$

Nuffield Orthopaedic Centre, Oxford University Hospitals NHS Trust, Oxford OX3 7HE, UK

e-mail: dominique.rothenfluh@mac.com
$\left(\Delta \mathrm{PILL} \geq 10^{\circ}\right.$ ) alignment according to pelvic incidencelumbar lordosis mismatch. In type A spino-pelvic alignment, $25.5 \%$ of patients underwent revision surgery for adjacent segment disease, whereas $78.3 \%$ of patients classified as type B alignment had revision surgery. Classification of patients into type A and B alignments yields a sensitivity for predicting adjacent segment disease of $71 \%$, a specificity of $81 \%$ and an odds ratio of 10.6.

Conclusion In degenerative disease of the lumbar spine a high pelvic incidence with diminished lumbar lordosis seems to predispose to adjacent segment disease. Patients with such pelvic incidence-lumbar lordosis mismatch exhibit a 10-times higher risk for undergoing revision surgery than controls if sagittal malalignment is maintained after lumbar fusion surgery.

Keywords Adjacent segment disease - Lumbar spinal fusion - Spino-pelvic alignment · Pelvic incidence-lumbar lordosis mismatch

\section{Introduction}

Spinal fusion is a common treatment for degenerative disorders of the lumbar spine providing adequate clinical results in terms of pain relief and high fusion rates [1]. However, spinal fusion has been associated with adjacent segment degeneration as a potential long-term sequel $[2,3]$. A recent meta-analysis reported a radiographic prevalence of adjacent segment degeneration from 4.8 to $92.2 \%$ [3]. Radiographic adjacent segment degeneration is poorly defined and does not correlate very well with symptomatic adjacent segment degeneration [4], which has also been termed adjacent segment disease [5]. In a study by Cheh et al. [6], $43 \%$ of patients showed radiographic 
adjacent segment degeneration, and $24 \%$ symptomatic adjacent segment disease, whereas $6.3 \%$ had clinical signs without radiographic evidence. The reported rates for adjacent segment disease in the literature range from 2.6 to $30.3 \%[2,6,7]$. Throughout the present article, the term adjacent segment disease is used for symptomatic adjacent segment degeneration.

Based on the rates of occurrence, adjacent segment degeneration and disease have a significant clinical impact and numerous studies have aimed at identifying risk factors. It has been indicated that patient factors such as age, gender, obesity, pre-existing degeneration, and facet tropism may contribute to adjacent segment degeneration $[2$, 7-9]. Lee et al. [7] reported in their study of 1,069 patients after lumbosacral fusion that mainly pre-existing degeneration of the facet joints may be a predisposing factor. Other authors have attributed adjacent segment degeneration and disease to surgical factors, such as the numbers of segments fused [10], increased postoperative disc height [11], or a low postoperative lordotic angle [12] with often conflicting findings among the different studies. However, risk factors which have relatively clear implications on subsequent surgical management have not been identified.

It has been suggested before that sagittal alignment may contribute to adjacent segment degeneration. Kumar et al. [13] concluded in their study that patients with a normal postoperative $\mathrm{C} 7$ plumbline and sacral inclination had the lowest risk of adjacent segment degeneration. A relationship between adjacent segment degeneration after lumbar fusion and spino-pelvic parameters, such as lumbar lordosis, pelvic incidence, pelvic tilt, and sacral slope has never been described, however. Legaye et al. [14] and DuvalBeaupère et al. [15] described a chain of correlations between positional parameters of upright posture and pelvic incidence. Boulay et al. [16] demonstrated an equation for predicting lumbar lordosis based on pelvic incidence and explained that within confidence limits the standing position is within the conditions of an economic posture and that out of confidence limits, the adaptation potential of the spine and pelvis is exceeded and may evoke pathological positions and loading patterns. Pelvic incidence has subsequently been generally acknowledged as a predictor of the amount of lumbar lordosis required to assume a balanced sagittal posture $[15,17,18]$. As the relationship between pelvic incidence as a morphologic parameter and lumbar lordosis seems important for the sagittal profile of the spine, it may also account for different loading patterns in the lumbar spine which may be relevant for the development of adjacent segment degeneration and disease.

In the present study, patients that underwent revision surgery for symptomatic adjacent segment disease were compared to a control group, to investigate whether differences in spino-pelvic parameters and especially spino-pelvic alignment could be associated with a higher risk for developing adjacent segment disease.

\section{Materials and methods}

For analysis of the relation of spino-pelvic parameters and adjacent segment disease, a retrospective case-control study was carried out. Approval of the research ethics committee of the state of Zurich has been granted for noninvasive, retrospective studies using patient data only. Patients were included into the adjacent segment disease group (ASDis) if they underwent primary lumbar fusion of one, two, or three segments between L2 and S1 and had surgery for symptomatic adjacent segment disease during follow-up. Indications for surgery were degenerative lumbar spondylosis or spondylolisthesis with leg pain or claudication. Patients that lacked complete preoperative radiographic documentation consisting of plain films and MRI, which had prior spine surgery or showed lumbar deformity, such as degenerative scoliosis or isthmic spondylolisthesis were excluded from further analysis. A control group (CTRL) was randomly selected out of the patient pool of lumbar fusion procedures that met the above inclusion criteria except for revision surgery to be similar in the distribution of age and gender to the ASDis group with the same exclusion criteria. In addition, patients were included in the CTRL group only if they had a minimal follow-up of 5 years, no signs of symptomatic adjacent segment disease upon last follow-up, similar distribution of levels and number of segments fused and comparable degree of disc degeneration in the prospective adjacent segment before the surgical procedure as assessed on MRI. All patients had standing radiographs of the lumbar spine with inclusion of the femoral heads; whole spine radiographs were not available.

In total, 84 patients were included which demonstrated adequate follow-up and radiographic documentation with preoperative MRI of the lumbar spine. All included patients had a posterolateral instrumented fusion with pedicle screws and showed union during follow-up. Fortyfive patients demonstrated adjacent segment disease (ASDis group), all of which were in the epifusional segments, and underwent revision surgery after a mean of 49 months (7-125 months). The average follow-up after the initial surgery was 71 months (15-149 months). The control group consists of 39 patients with a mean follow-up of 84 months (61-142 months). The average body mass index (BMI) in the ASDis group is $26.2 \pm 4.4$ vs. $26.8 \pm 4.0$ in the CTRL group. Details on the patients' characteristics are given in Table 1 . The patients in the control group were selected to be similar in the distribution of the levels treated and number of segments fused 
Table 1 Patients

\begin{tabular}{lll}
\hline$n=84$ & ASDis $(n=45)$ & CTRL $(n=39)$ \\
\hline Follow-up (mean) & $\begin{array}{c}\text { (1 months } \\
(15-149)\end{array}$ & $\begin{array}{c}\text { (61-142) } \\
\text { Agenths }\end{array}$ \\
Sex & 58 years $(34-81)$ & 64 years $(45-83)$ \\
Body mass index (BMI) & $26.2 \pm 4.4$ & 27 female \\
No. of segments L2-S1 & $1: 19 \mathrm{pts}$ & $1: 18 \mathrm{pts}$ \\
& $2: 17 \mathrm{pts}$ & $2: 15 \mathrm{pts}$ \\
& $3: 9 \mathrm{pts}$ & $3: 6 \mathrm{pts}$ \\
Revision for ASD & 49 months $(7-125)$ & \\
$\quad$ (mean) & & \\
\hline
\end{tabular}

Table 2 Levels and number of segments fused

\begin{tabular}{lll}
\hline$n=84$ & ASDis $(n=45)$ & CTRL $(n=39)$ \\
\hline L2-L5 (3) & 6 & 4 \\
L3-L4 (1) & 2 & 2 \\
L3-L5 (2) & 9 & 7 \\
L3-S1 (3) & 3 & 2 \\
L4-L5 (1) & 12 & 10 \\
L4-S1 (2) & 8 & 8 \\
L5-S1 (1) & 5 & 6 \\
\hline
\end{tabular}

(Table 2). To rule out that the ASDis group had more preoperative degenerative changes of the lumbar spine resulting in adjacent segment disease, the patients in the control group were selected to have similar preoperative grade of disc degeneration on MRI (Table 3). Selecting similar groups in the distribution of their matching variables was carried out before measurements were performed on radiographs to minimize selection bias.

Analysis of radiographs consisted of measurements of spino-pelvic parameters, such as pelvic incidence (PI), sacral slope (SS), pelvic tilt (PT), lumbar lordosis (LL), and L1 plumb line. Figure 1 depicts how measurements were taken. Measurements were taken on the preoperative radiographs and at last follow-up in the CTRL group or before revision surgery in the ASDis group. LL was measured from L1-L5 and L1-S1 for assessment of their sensitivity as described below. In addition, measurements of lordosis of the segments to be fused and the postoperative fusion angle (fusion angle) as well as the pre- and postoperative angles of the epifusional (upper AS angle) and subjacent segments (lower AS angle) were measured by sagittal Cobb measurements in lateral radiographs. PILL mismatch ( $\triangle \mathrm{PILL})$ was calculated as the difference between PI and LL $(\Delta \mathrm{PILL}=\mathrm{PI}-\mathrm{LL})$. Measurements were performed by two raters and Bland-Altman analysis performed to indicate the accuracy of measurement [19].
Table 3 Preoperative Pfirrmann grade of the adjacent disc

\begin{tabular}{lll}
\hline Pfirrmann grade & ASDis $(n=45)$ & CTRL $(n=39)$ \\
\hline 1 & 2 & 3 \\
2 & 19 & 15 \\
3 & 16 & 13 \\
4 & 5 & 6 \\
5 & 2 & 2 \\
\hline
\end{tabular}

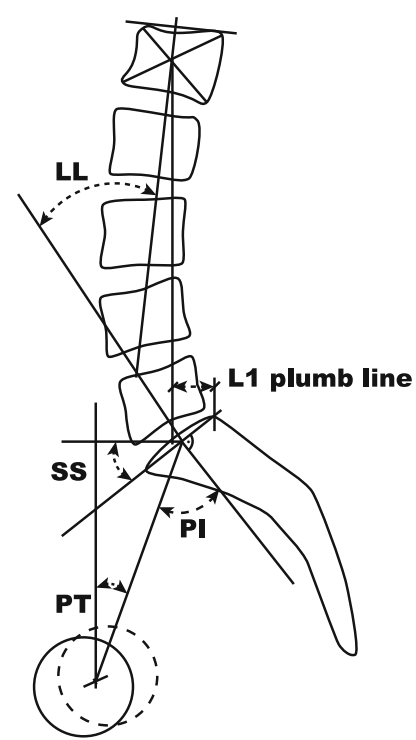

Fig. 1 Measurements were carried out as shown: lumbar lordosis is measured as the sagittal Cobb angle between the superior endplate of L1 and the sacral plateau S1. Pelvic incidence is the angle of a line perpendicular to the $\mathrm{S} 1$ endplate at its midpoint and a line connecting to the midpoint of the line connecting the centers of the femoral heads. Pelvic tilt is measured as the angle between a vertical line trough the midpoint of the centers of the femoral head and a line connecting to the midpoint of the endplate of S1. Finally, sacral slope is measured as the angle between a horizontal line and the endplate of $\mathrm{S} 1$

For subsequent analyses, the mean of the two measurements was used. The inter-rater bias for PI, LL, and $\triangle \mathrm{PILL}$ was $0.78 \pm 2.2,0.34 \pm 1.45$, and $0.56 \pm 1.89$, indicating less measurement accuracy for PI. The $95 \%$ limits of agreement were calculated to range from 5.1 to $-3.5,3.2$ to -2.5 , and 4.3 to -3.1 , respectively. Disc degeneration was graded on preoperative MRI of the lumbar spine according to Pfirrmann et al. [20]. Degeneration of facet joints could not be fully assessed as not all MRI included axial images of all segments of the lumbar spine and, therefore, had to be excluded.

\section{Statistics}

Analysis of all data was carried out using JMP 9.0 for Mac (SAS Institute Inc., Cary, North Carolina, USA). Normality 
of distribution was checked by the Shapiro-Wilk test. For the variables lumbar lordosis $(p=0.56)$, pelvic incidence ( $p=0.82)$, pelvic tilt $(p=0.71)$, sacral slope $(p=0.09)$, $\Delta$ PILL $(p=0.92)$, and PT/SS $(p=0.06)$, a normal distribution could be assumed and therefore parametric testing used. A dependent and independent $t$ test statistic was employed for intra-group and inter-group comparisons, respectively. For the variables L1 plumb line, fusion angle, upper AS angle, and lower AS angle, a normal distribution could not be confirmed. Intra-group comparisons were carried out by the Wilcoxon signed-rank test and between group comparisons by the Wilcoxon rank-sum/MannWhitney tests. All $p$ values reported are 2-tailed and considered significant if $p<0.05$. Only significant $p$ values are reported. Classification was performed using logistic regression and ROC curve analysis. For ROC curve graphing, the pROC library for the $\mathrm{R}$ statistics package was used [21].

\section{Results}

Comparison of the pre- and postoperative values within the groups showed significant differences in the ASDis as well as CTRL group for lumbar lordosis (ASDis $p=0.012$ and CTRL $p=0.027$ ) and pelvic incidence (ASDis $p=0.017$ and CTRL $p=0.002$ ), for pelvic tilt only in the CTRL group $(p=0.011)$ and for sacral slope only in the ASDis group $(p=0.011)$. The differences between pre- and postoperative measurements are within a few degrees and can be regarded as largely unchanged although significant, as the differences are within the limits of measurement accuracy as indicated by the Bland-Altman analysis above. All measurement results are summarized in Table 4. Fusion did not change the preoperative lordosis angle of the segments to be fused in both groups (ASDis: $26.5 \pm 11.3^{\circ}$ vs. $24.4 \pm 9.3^{\circ}, p=0.162$; CTRL:23.6 \pm $12.6^{\circ}$ vs. $\left.22.7 \pm 8.6^{\circ}, p=0.535\right)$. Likewise, the segmental angle in the upper and lower adjacent segments did not change from pre- to postoperative in both groups, indicating that they unlikely contribute to adjacent segment degeneration and disease. Subsequent analyses focused on the postoperative measurements only to link postfusion alignment to adjacent segment disease.

Comparison of the radiographic parameters between the groups reveals that patients in the ASDis group have a significantly higher pelvic incidence than in the CTRL group $\left(60.9 \pm 10.0^{\circ}\right.$ vs. $\left.51.7 \pm 10.4, p=0.001\right)$ and correspondingly also a higher pelvic tilt $\left(22.4 \pm 7.0^{\circ}\right.$ vs. $\left.18.8 \pm 6.5^{\circ}, p=0.012\right)$ and slightly higher sacral slope $\left(37.2 \pm 8.0^{\circ}\right.$ vs. $\left.35.3 \pm 7.7^{\circ}, p=0.004\right)$. In addition, lumbar lordosis was significantly lower in the ASDis group compared to the CTRL group (48.1 $\pm 12.5^{\circ}$ vs. $53.8 \pm 10.8^{\circ}, p=0.012$ ). To assess alignment, pelvic incidence was put into relation to lumbar lordosis by calculating the difference between pelvic incidence and lumbar lordosis measured to S1 ( $\triangle \mathrm{PILL}=\mathrm{PI}-\mathrm{LL})$ following the notion that lumbar lordosis should more or less match pelvic incidence. $\triangle$ PILL showed a large significant difference in spino-pelvic alignment between the ASDis and CTRL group (12.5 $\pm 16.7^{\circ}$ vs. $3.4 \pm 12.1^{\circ}$, $p=0.001$ ), indicating that it may be a potentially contributing factor to adjacent segment disease. It was further found that the L1 plumb line, measured as the horizontal distance between the posterior endplate of $\mathrm{S} 1$ and the plumb line from the center of L1, was slightly shifted anteriorly in the ASDis group (39.4 $\pm 68.2 \mathrm{~mm}$ vs. $23.6 \pm 61.4 \mathrm{~mm}$, n.s.), which corresponds to the lower lordosis in this group compared to the CTRL group. Despite the large difference for the L1 plumb line between ASDis and CTRL, it is not significant, which may be explained by the large distribution of values as indicated by the standard deviation. In addition, the relationship of the L1 plumb line to global balance is not defined and conclusions on the overall balance can therefore not be made from this measure.
Table 4 Radiographic measurements

\begin{tabular}{|c|c|c|c|c|}
\hline & \multicolumn{2}{|c|}{ ASDis $(n=45)$; mean \pm SD } & \multicolumn{2}{|c|}{ CTRL $(n=39)$; mean \pm SD } \\
\hline & Preop & Postop & Preop & Postop \\
\hline Lumbar lordosis L1-L5 $\left(^{\circ}\right)$ & $38.5 \pm 11.1$ & $39.6 \pm 11.2$ & $43.7 \pm 10.2$ & $41.6 \pm 10.5$ \\
\hline Lumbar lordosis L1-S1 $\left(^{\circ}\right)$ & $48.8 \pm 13.5$ & $48.1 \pm 12.5$ & $54.6 \pm 9.6$ & $53.8 \pm 10.8$ \\
\hline Pelvic incidence $\left(^{\circ}\right)$ & $60.9 \pm 10.0$ & $59.5 \pm 10.1$ & $51.7 \pm 10.4$ & $53.9 \pm 10.5$ \\
\hline Pelvic tilt $\left({ }^{\circ}\right)$ & $22.2 \pm 7.3$ & $22.4 \pm 7.0$ & $16.8 \pm 6.8$ & $18.6 \pm 6.5$ \\
\hline Sacral slope $\left(^{\circ}\right)$ & $36.2 \pm 8.4$ & $37.8 \pm 8.0$ & $34.9 \pm 7.6$ & $35.3 \pm 7.7$ \\
\hline L1 plumb line (mm) & $42.7 \pm 76.1$ & $39.4 \pm 68.2$ & $21.0 \pm 82.4$ & $23.6 \pm 61.4$ \\
\hline Fusion angle $\left({ }^{\circ}\right)$ & $23.6 \pm 12.6$ & $22.7 \pm 8.6$ & $26.5 \pm 11.3$ & $24.4 \pm 9.3$ \\
\hline Upper AS angle $\left(^{\circ}\right)$ & $8.8 \pm 4.8$ & $8.6 \pm 4.1$ & $10.4 \pm 3.3$ & $9.4 \pm 3.4$ \\
\hline Lower AS angle $\left({ }^{\circ}\right)$ & $11.9 \pm 6.0$ & $10.9 \pm 6.9$ & $9.6 \pm 6.7$ & $9.8 \pm 6.3$ \\
\hline
\end{tabular}


Classification of alignment according to PILL mismatch in relation to adjacent segment disease

Logistic regression revealed that $\triangle \mathrm{PILL}$ as a measure of spino-pelvic alignment can be used to classify patients into the two groups (Chi-square $=12.5, p<0.0001$ ), while other radiographic factors such as pelvic incidence or lumbar lordosis alone were significant but showed less sensitivity on subsequent analyses (Chi-square $=11.82$, $p<0.0001$ and Chi square $=6.53, p=0.0106$, respectively). Sacral slope did not show any discrimination between the two groups (Chi-square $=3.06, p=0.18$ ). For $\triangle$ PILL a cutoff value of $9.8^{\circ}$ was determined to discriminate between the two groups with the highest sensitivity and specificity by ROC analysis with an area under the curve $\mathrm{AUC}=0.73 \quad(95 \%$ confidence interval 0.62-0.84; Fig. 2). The same analysis for measurements of LL from $\mathrm{L} 1$ to $\mathrm{L} 5$ reveals a cutoff value of $15.2^{\circ}$ and AUC of the ROC curve equals 0.87 . For scientific purposes, this measurement was used for classification in a subsequent biomechanical study [22]; however, for clinical use, $\triangle$ PILL used in this study signifies pelvic incidence-lumbar lordosis mismatch with LL measured from L1 to S1 as internationally widely agreed. Based on the cutoff value of $\triangle \mathrm{PILL}$ of $10^{\circ}$, patients were classified into two types of alignment according to pelvic incidence-lumbar lordosis (PILL) mismatch. In type A alignment, the difference between pelvic incidence and lumbar lordosis $(\triangle \mathrm{PILL})$ is below $10^{\circ}$

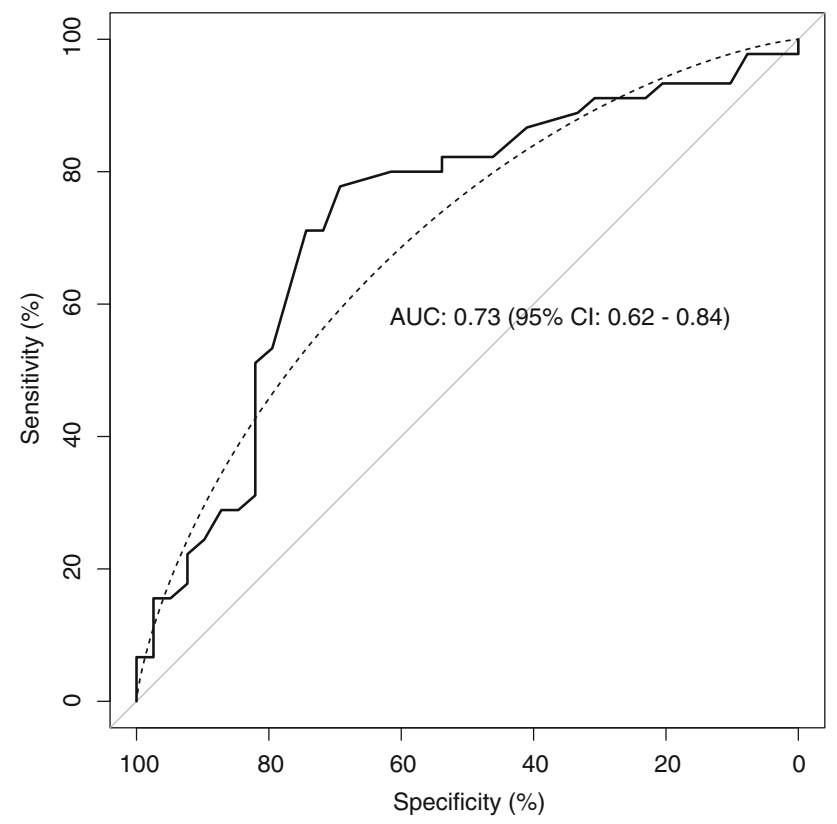

Fig. 2 Logistic regression and ROC analysis revealed a cutoff value for $\triangle \mathrm{PILL}$ of $9.8^{\circ}$ at which classification according to $\triangle \mathrm{PILL}$ yields a sensitivity of $71 \%$ and specificity of $81 \%$. The area under the curve AUC is 0.73 with a $95 \%$ confidence interval from 0.62 to 0.84 and in type $\mathrm{B}$ alignment, $\triangle$ PILL is above or equal to $10^{\circ}$. Forty-one patients were classified as type A alignment, 29 belonged to the CTRL and 12 to the ASDis group. In type B alignment, 35 out of 43 patients belong to the ASDis and only 8 to the CTRL group. In type A alignment, $25.5 \%$ of patients underwent revision surgery for adjacent segment disease, whereas $78.3 \%$ of patients classified as type B alignment had revision surgery. Classification of patients according to $\triangle \mathrm{PILL}<10^{\circ}$ yields a sensitivity for predicting adjacent segment disease of $71 \%$ with a specificity of $81 \%$. The odds ratio as an estimate of the relative risk for developing adjacent segment disease is 10.6, indicating a 10-times higher risk for patients with a type B alignment and a high degree of PILL mismatch. The characteristics of patients according to their classification are given in Table 5. Comparison of ASDis and CTRL within type A and type $\mathrm{B}$ alignments, i.e., false compared to true negatives and false compared to true positives with reference to adjacent segment disease, does not show any significant differences. On the other hand, comparison between type A and type B not distinguishing between ASDis and CTRL showed significant differences for all variables except for values of L1 plumb line, indicating two distinct morphologies of spino-pelvic alignment (Table 5, Fig. 3). In type B alignment, the pelvis assumes a compensatory position with increased pelvic tilt as indicated by the ratio PT/SS which was different between type $\mathrm{A}$ and $\mathrm{B}$ alignments $(0.53 \pm 0.19$ vs. $0.67 \pm 0.23, p=0.004)$.

\section{Discussion}

This investigation was designed and the control group selected to be similar in the distribution of the matching variables to investigate spino-pelvic alignment in relation to adjacent segment disease irrespective of preoperative degenerative changes, levels and number of segments fused. In accordance with the previously established relationship of pelvic incidence and lumbar lordosis and the notion that the two should match within certain limits [15, $17,18]$, the difference $\triangle$ PILL was calculated as a measure of spino-pelvic alignment. $\triangle$ PILL was significantly different in the ASDis and CTRL group and could be shown to be useful for classifying patients by logistic regression at a cutoff value of $10^{\circ}$. Classification of PILL mismatch into type $\mathrm{A}\left(\Delta \mathrm{PILL}<10^{\circ}\right)$ and type $\mathrm{B}\left(\Delta \mathrm{PILL} \geq 10^{\circ}\right)$ shows adequate sensitivity and reliability for predicting the risk of adjacent segment disease and patients with $\triangle$ PILL greater than $10^{\circ}$ have a 10 -times higher risk of developing adjacent segment disease. The relationship between local alignment described as PILL mismatch and global sagittal balance, which may be a potential contributing factor, could not be assessed due to the retrospective nature of the study as 
Table 5 Classification of spino-pelvic alignment into type A and B according to $\triangle \mathrm{PILL}$

\begin{tabular}{|c|c|c|c|c|c|c|}
\hline \multirow[t]{2}{*}{ Postoperative } & \multicolumn{3}{|c|}{$\begin{array}{l}\text { Type A: } \triangle \text { PILL }<10^{\circ} \\
\text { mean } \pm \text { SD }\end{array}$} & \multicolumn{3}{|c|}{$\begin{array}{l}\text { Type } B: \Delta \text { PILL } \geq 10^{\circ} \\
\text { mean } \pm \mathrm{SD}\end{array}$} \\
\hline & ASDis $(n=12)$ & CTRL $(n=29)$ & Combined & ASDis $(n=35)$ & CTRL $(n=8)$ & Combined \\
\hline$\Delta$ PILL $(\mathrm{PI}-\mathrm{LL})\left({ }^{\circ}\right)$ & $0.75 \pm 6.5$ & $-2.4 \pm 8.1$ & $-1.5 \pm 7.7$ & $19.3 \pm 10.1$ & $18.1 \pm 6.6$ & $19.0 \pm 9.3$ \\
\hline Lordosis $\left({ }^{\circ}\right)$ & $56.6 \pm 8.7$ & $50.5 \pm 9.1$ & $52.3 \pm 9.3$ & $44.3 \pm 12.1$ & $42.7 \pm 9.6$ & $43.9 \pm 11.4$ \\
\hline Pelvic incidence $\left(^{\circ}\right)$ & $54.6 \pm 8.7$ & $50.8 \pm 10.7$ & $51.7 \pm 10.3$ & $63.0 \pm 9.7$ & $58.0 \pm 5.9$ & $62.3 \pm 9.4$ \\
\hline Pelvic tilt $\left(^{\circ}\right)$ & $17.5 \pm 5.7$ & $16.3 \pm 5.5$ & $16.7 \pm 5.5$ & $24.3 \pm 6.6$ & $24.4 \pm 5.1$ & $24.3 \pm 6.2$ \\
\hline Sacral slope $\left(^{\circ}\right)$ & $39.9 \pm 8.4$ & $34.0 \pm 7.5$ & $35.6 \pm 8.1$ & $37.1 \pm 7.8$ & $38.5 \pm 7.6$ & $37.5 \pm 7.7$ \\
\hline $\mathrm{PT} / \mathrm{SS}$ & $0.63 \pm 0.17$ & $0.49 \pm 0.19$ & $0.53 \pm 0.19$ & $0.70 \pm 0.26$ & $0.56 \pm 0.18$ & $0.67 \pm 0.23$ \\
\hline L1 plumb line $(\mathrm{mm})$ & $49.1 \pm 62.0$ & $19.7 \pm 85.6$ & $26.4 \pm 71.2$ & $44.5 \pm 60.0$ & $9.5 \pm 43.1$ & $41.7 \pm 57.7$ \\
\hline
\end{tabular}

type A

$\triangle$ PILL $<10^{\circ}$

type B

$\triangle$ PILL $\geq 10^{\circ}$
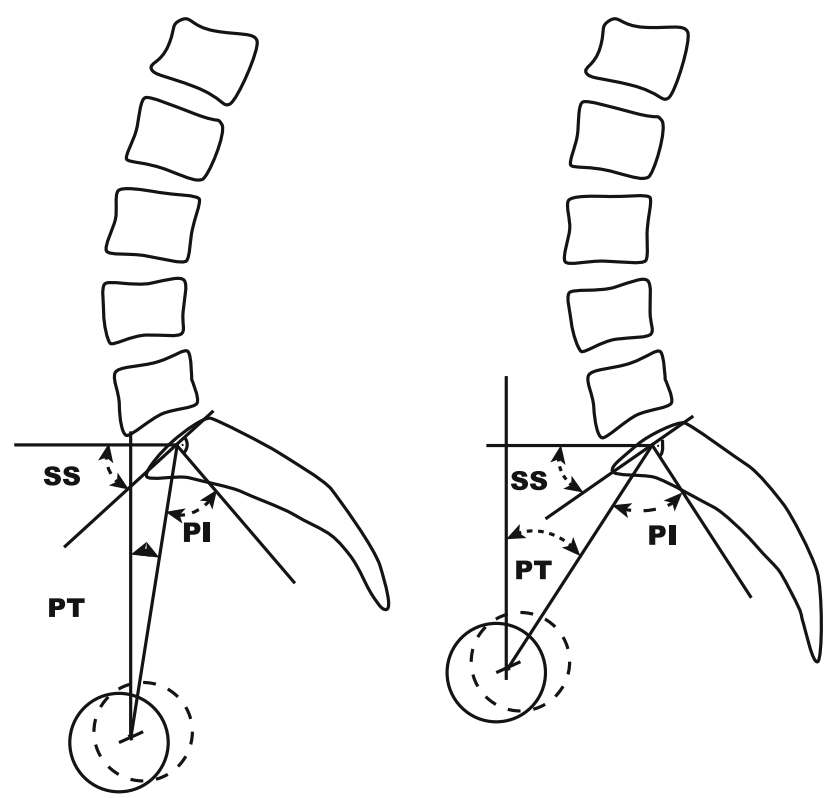

Fig. 3 The two distinct morphologies of pelvic incidence-lumbar lordosis mismatch are shown. In a type A alignment, pelvic incidence is in the normal range with a corresponding lumbar lordosis and balanced lumbar spine, consequently $\triangle \mathrm{PILL}$ is below $10^{\circ}$. In a type $\mathrm{B}$ alignment, the pelvic incidence is greater than in type A with slight pelvic back tilt. If this pelvic morphology is not paralleled by a corresponding lumbar lordosis, the resulting mismatch predisposes to a higher risk for adjacent segment disease

whole spine radiographs were not obtained routinely for investigating the lumbar spine and were therefore not available for analysis.

The etiology of adjacent segment degeneration and disease has been related to biomechanical alterations following spinal fusion [23-26] or regarded simply as progression of the degenerative process not influenced by the fusion procedure $[12,27]$. As such it is likely to be multifactorial and disc degeneration and therefore degeneration of the motion segment might be related to genetic influences and has been associated with for example polymorphisms of the vitamin D receptor and collagen IX genes [28]. Studies on altered kinematics and biomechanics after lumbar spinal fusion have not focused on spino-pelvic alignment, but investigated hypermobility and increased loads in the adjacent segment $[24,26]$. Our findings indicate that PILL mismatch describes different morphologies with expected consequences on spinal biomechanics (Fig. 3). Our data show that in some cases a high pelvic incidence is not matched by a correspondingly high lordosis as would be expected in a balanced spine $[29,30]$ and therefore optimal congruence between pelvic and spinal parameters for spinal balancing and for an economical posture not present [31]. It seems likely and is expected from a biomechanical point of view that differences in $\triangle \mathrm{PILL}$ as a measure of spino-pelvic alignment have consequences on the load patterns in the lumbar spine and even more so in the adjacent segment after spinal fusions, which is in accordance with findings of previous studies [13, 32]. Whether adjacent segment disease was evoked by the fusion procedure or whether more degenerative changes would have been seen in a non-fusion group over time as a result of a type B alignment remains to be investigated. However, a subsequent modeling study has shown that segmental joint reaction forces acting on the motion segment are unfavorable in patients with PILL mismatch before fusion [22], indicating that alignment may contribute to the natural history. The study of segmental biomechanics in the background of fusion should therefore not be decoupled from alignment and the resulting forces thereof.

The relevance of pelvic incidence-lumbar lordosis mismatch in adult deformity has just very recently been reported in a study by authors of the International Spine Study Group [33]. In their analysis of 492 patients with adult spinal deformity in a prospective database, they identified the sagittal vertical axis (SVA), pelvic tilt, and PILL mismatch to correlate with disability and quality of life as defined by an Oswestry Disability Index (ODI) of 
more than 40 . PILL mismatch of more than $11^{\circ}$ was found to have the strongest correlation with disability, but also correlations with the SVA and pelvic tilt. Therefore, PILL mismatch of $11^{\circ}$ or more indicated that patients are either likely to be unbalanced or compensating. Their values compare favorably to our cutoff value of $10^{\circ}$ for adjacent segment disease. Schwab et al. [33] concluded from their analysis that PILL mismatch is an intrinsic part of the adult deformity and restoration of PILL mismatch should be the primary objective in the surgical management of adult deformity. Their study indicates that about a third of patients with PILL mismatch of more than $11^{\circ}$ did not have global sagittal imbalance as defined by an SVA greater than $47 \mathrm{~mm}$ [33]. While in the present study global sagittal balance could not be measured, the patients reported here were treated for lumbar degeneration and not for global sagittal imbalance. PILL mismatch brings the deformity world into the degenerative lumbar spine and indicates an intrinsic deformity whereby global sagittal imbalance does not necessarily have to be present. If PILL mismatch and, therefore, the intrinsic deformity of the degenerative spine is not addressed in a fusion procedure and fusion is carried out by maintaining the same extent of lordosis, then these patients are at higher risk for adjacent segment disease as demonstrated here.

Acknowledgments DAR designed and planned the study, acquired and analyzed all data and wrote the manuscript, all other authors contributed in data acquisition and analysis as well as manuscript preparation.

\section{Conflict of interest None.}

\section{References}

1. Fritzell P, Hagg O, Wessberg P, Nordwall A, Swedish Lumbar Spine Study G (2001) 2001 Volvo Award Winner in Clinical Studies: Lumbar fusion versus nonsurgical treatment for chronic low back pain: a multicenter randomized controlled trial from the Swedish Lumbar Spine Study Group. Spine (Phila Pa 1976) 26:2521-2532 (discussion 2532-2524)

2. Park P, Garton HJ, Gala VC, Hoff JT, McGillicuddy JE (2004) Adjacent segment disease after lumbar or lumbosacral fusion: review of the literature. Spine 29:1938-1944

3. Xia XP, Chen HL, Cheng HB (2013) Prevalence of adjacent segment degeneration after spine surgery: a systematic review and meta-analysis. Spine (Phila Pa 1976) 38:597-608

4. Min J-H, Jang J-S, Bj Jung, Lee HY, Choi W-C, Shim CS, Choi G, Lee S-H (2008) The clinical characteristics and risk factors for the adjacent segment degeneration in instrumented lumbar fusion. J Spinal Disord Tech 21:305-309

5. Harrop JS, Youssef JA, Maltenfort M, Vorwald P, Jabbour P, Bono CM, Goldfarb N, Vaccaro AR, Hilibrand AS (2008) Lumbar adjacent segment degeneration and disease after arthrodesis and total disc arthroplasty. Spine 33:1701-1707

6. Cheh G, Bridwell KH, Lenke LG, Buchowski JM, Daubs MD, Kim Y, Baldus C (2007) Adjacent segment disease following lumbar/thoracolumbar fusion with pedicle screw instrumentation: a minimum 5-year follow-up. Spine 32:2253-2257

7. Lee CS, Hwang CJ, Lee S-W, Ahn Y-J, Kim Y-T, Lee D-H, Lee MY (2009) Risk factors for adjacent segment disease after lumbar fusion. Eur Spine J 18:1637-1643

8. Anandjiwala J, Seo JY, Ha KY, Oh IS, Shin DC (2011) Adjacent segment degeneration after instrumented posterolateral lumbar fusion: a prospective cohort study with a minimum five-year follow-up. Eur Spine J 20:1951-1960

9. Aota Y, Kumano K, Hirabayashi S (1995) Postfusion instability at the adjacent segments after rigid pedicle screw fixation for degenerative lumbar spinal disorders. J Spinal Disord 8:464-473

10. Gillet $P$ (2003) The fate of the adjacent motion segments after lumbar fusion. J Spinal Disord Tech 16:338-345

11. Kaito T, Hosono N, Mukai Y, Makino T, Fuji T, Yonenobu K (2010) Induction of early degeneration of the adjacent segment after posterior lumbar interbody fusion by excessive distraction of lumbar disc space. J Neurosurg Spine 12:671-679

12. Kim KH, Lee S-H, Shim CS, Lee DY, Park HS, Pan W-J, Lee HY (2010) Adjacent segment disease after interbody fusion and pedicle screw fixations for isolated 14-15 spondylolisthesis: a minimum five-year follow-up. Spine 35:625-634

13. Kumar MN, Baklanov A, Chopin D (2001) Correlation between sagittal plane changes and adjacent segment degeneration following lumbar spine fusion. Eur Spine J 10:314-319

14. Legaye J, Duval-Beaupere G, Hecquet J, Marty C (1998) Pelvic incidence: a fundamental pelvic parameter for three-dimensional regulation of spinal sagittal curves. Eur Spine J 7:99-103

15. Duval-Beaupere G, Schmidt C, Cosson P (1992) A Barycentremetric study of the sagittal shape of spine and pelvis: the conditions required for an economic standing position. Ann Biomed Eng 20:451-462

16. Boulay C, Tardieu C, Hecquet J, Benaim C, Mouilleseaux B, Marty C, Prat-Pradal D, Legaye J, Duval-Beaupere G, Pélissier J (2005) Sagittal alignment of spine and pelvis regulated by pelvic incidence: standard values and prediction of lordosis. Eur Spine J $15: 415-422$

17. Roussouly P, Gollogly S, Berthonnaud E, Dimnet J (2005) Classification of the normal variation in the sagittal alignment of the human lumbar spine and pelvis in the standing position. Spine 30:346-353

18. Schwab F, Patel A, Ungar B, Farcy JP, Lafage V (2010) Adult spinal deformity-postoperative standing imbalance: how much can you tolerate? An overview of key parameters in assessing alignment and planning corrective surgery. Spine 35:2224-2231

19. Bland JM, Altman DG (1986) Statistical methods for assessing agreement between two methods of clinical measurement. Lancet $1: 307-310$

20. Pfirrmann CW, Metzdorf A, Zanetti M, Hodler J, Boos N (2001) Magnetic resonance classification of lumbar intervertebral disc degeneration. Spine 26:1873-1878

21. Robin X, Turck N, Hainard A, Tiberti N, Lisacek F, Sanchez JC, Muller M (2011) pROC: an open-source package for $\mathrm{R}$ and $\mathrm{S}+$ to analyze and compare ROC curves. BMC Bioinformatics $12: 77$

22. Senteler M, Weisse B, Snedeker JG, Rothenfluh DA (2014) Pelvic incidence-lumbar lordosis mismatch results in increased segmental loads in the unfused and fused lumbar spine. Eur Spine J. doi:10.1007/s00586-013-3132-7

23. Axelsson P, Johnsson R, Strömqvist B (1997) The spondylolytic vertebra and its adjacent segment. Mobility measured before and after posterolateral fusion. Spine 22:414-417

24. Bastian L, Lange U, Knop C, Tusch G, Blauth M (2001) Evaluation of the mobility of adjacent segments after posterior thoracolumbar fixation: a biomechanical study. Eur Spine J $10: 295-300$ 
25. Ekman P, Möller H, Shalabi A, Yu YX, Hedlund R (2009) A prospective randomised study on the long-term effect of lumbar fusion on adjacent disc degeneration. Eur Spine J 18:1175-1186

26. Weinhoffer SL, Guyer RD, Herbert M, Griffith SL (1995) Intradiscal pressure measurements above an instrumented fusion. A cadaveric study. Spine 20:526-531

27. Ruberté LM, Natarajan RN, Andersson GB (2009) Influence of single-level lumbar degenerative disc disease on the behavior of the adjacent segments-a finite element model study. J Biomech 42:341-348

28. Chan D, Song Y, Sham P, Cheung KM (2006) Genetics of disc degeneration. Eur Spine J 15(Suppl 3):S317-S325

29. Barrey C, Roussouly P, Perrin G (2011) Sagittal balance disorders in severe degenerative spine. Can we identify the compensatory mechanisms? Eur Spine J 20(Suppl 5):626-633
30. Roussouly P, Pinheiro-Franco JL (2011) Biomechanical analysis of the spino-pelvic organization and adaptation in pathology. Eur Spine J 20(Suppl 5):609-618

31. Barrey C, Jund J, Noseda O, Roussouly P (2007) Sagittal balance of the pelvis-spine complex and lumbar degenerative diseases. A comparative study about 85 cases. Eur Spine J 16:1459-1467

32. Djurasovic MO, Carreon LY, Glassman SD, Dimar JR, Puno RM, Johnson JR (2008) Sagittal alignment as a risk factor for adjacent level degeneration: a case-control study. Orthopedics 31:546

33. Schwab FJ, Blondel B, Bess S, Hostin R, Shaffrey CI, Smith JS, Boachie-Adjei O, Burton DC, Akbarnia BA, Mundis GM, Ames CP, Hart RA, Farcy JP, Lafage V, International Spine Study G (2013) Radiographical spinopelvic parameters and disability in the setting of adult spinal deformity: a prospective multicenter analysis. Spine (Phila Pa 1976) 38:E803-E812 